\title{
XXX. Beiträge zur Kenntniss der Alunit- Jarositgruppe.
}

\author{
Von \\ W. F. Hillebrand in Washington und s. $\mathbf{I}$. Penfleld in New Haven, Conn.
}

(Mit 2 Textfiguren.)

Im Nachstehenden geben wir die Beschreibung zweier neuer Varietäten des Jarosits. Die eine derselben wurde aus Nevada von Ilerrn II. WV. Turner zur Bestimmung an das Laboratorium der Geol. Survey in Washington eingesendet; die andere stammt aus New Mexico und wurde von lJerrn J. II. Porter in Denver, Col., dem mineralogischen Laboratorium der Sheffield Sc. School zur Identificirung übergeben. Mit Ausnahme einer kleinen Verschiedenheit in der Färbung gleichen die beiden Vorkommen einander völlig. Das Mineral bildet winzige, isolirte, tafelige Krystalle, welche unter dem Mikroskope die Combination eines Rhomboëders mit gross ausgebildeter Basis erkennen lassen. Der Zufall fügte es, dass die beiden Autoren davon Kenntniss erhielten, dass sie gleichzeitig mit der Untersuchung zweier Mineralien derselben Gruppe beschäftigt waren, und so vereinigten sie sich, um ihre Resultate in nachstehender gemeinschaftlicher Publication niederzulegen.

\section{Natrojarosit.}

Das von Herrn Turner gesammelte Material stammt von der Ostseite des Soda-Springs Valley, am Wege von Sodaville nach der Vulcan Copper Mine. Es bildet ein schimmerndes Pulver, bestehend aus winzigen, vollkommen ausgebildeten Kryställchen vom Habitus wie Fig. 1; die meisten derselben zeigen jedoch nur das eine Rhomboëder $r$ an Stelle der beiden in der Figur abgebildeten.

Groth, Zeitschrift f. Krystallogr. XXXVI.
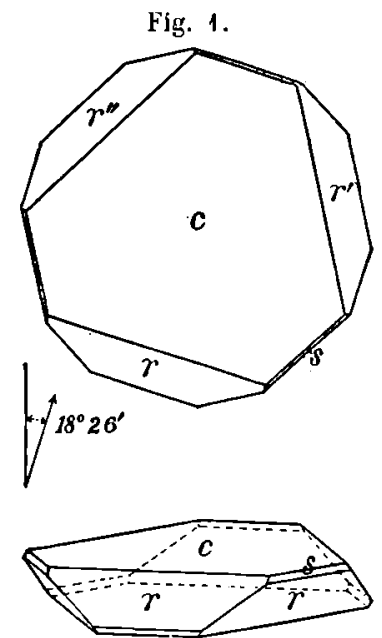

35 
Der grösste Krystall war $0,15 \mathrm{~mm}$ breit und $0,025 \mathrm{~mm}$ dick, die meisten Krystalle sind aber nur halb so gross. Trolz dieser geringen Dimensionen gelang es doch, die Winkol mit dem Reflexionsgoniometer zu messen; Schwierigkeiten bereitete nur der vicinale (harakter der Basisfläche.

Nach wiederholten Versuchen wurde ein Krystall gefunden, dessen Basis von hinlünglich guter Beschaffenheit war und der folgende Messungen gestattete:

Gemessen:

Gemessen: Berechnet:

$c: r(0001):(10 T 1)=51^{053^{*}} \quad c: s(0001):(02 \overline{2} 1)=68^{0} 42^{\prime} \quad \mathbf{6 8}^{0} 35^{\prime}$

$c: r^{\prime}(0001):(\overline{1} 101) \quad 5153 \quad c: s^{\prime}(0001):(\overline{2} 021) \quad 6848 \quad 6835$

$c: r^{\prime \prime}(0001):(0 T 11) \quad 5226 \quad r: r^{\prime}(10 T 1):(1101) \quad 86 \quad 5 \quad 8554$

Die Krystalle sind hexagonal-rhomboëdrisch. Der als Fundamentalwinkel angenommene Werth $51^{\circ} 53^{\prime}$, der ziemlich genau sein dürfte, führt auf das Axenverhältniss $a: c=1: 1,104$; dass dieses ziemlich der Wirklichkeit entspricht, ergiebt sich aus den Werthen für $c: s$ und $r: r$, welche nur wenig von den berechneten abweichen. Eine weitere Reihe von Messungen des Winkels $c: r$, welche zwar beträchtlich schwankten, ergaben Werthe um 520. Die Winkel $c: r$ und $r: r^{\prime}$ des gewöhnlichen Kaliumjarosits sind $55^{0} 16^{\prime}$ und $90^{\circ} 45^{\prime}$.

Unter dem Mikroskope zeigen die Krystalle normale optische Eigenschaften, die dickeren zeigen das schwarze Kreuz und den Beginn des ersten Interferenzringes, die Doppelbrechung jst negativ. Die Farbe einzelner Krystalle im durchfallenden Lichte ist goldgelb, während die Farbe einer grösseren Menge von Krystallen gelbbraun ist. Viele Krystalle zeigen auch zahlreiche braune Einschlüsse. Das erwähnte Glitzern des Materials entsteht durch Reflexion an den zahlreichen Basisflächen.

Das zur Analyse verwendete Material war so rein als möglich, doch konnten Krystalle mit den erwähnten braunen Einschlüssen nicht umgangen werden; auch fanden sich gelegentlich einige braune eisenreiche Partikel mit den Krystallen gemengt. Das spec. Gew. wurde bei $30,5^{\circ}$ C. zu 3,18 gefunden. Die Analyse (Hillebrand) ergab:

\begin{tabular}{|c|c|c|c|}
\hline \multirow[b]{2}{*}{$\mathrm{Fe}_{2} \mathrm{O}_{3}$} & \multicolumn{3}{|c|}{ Verhältniss: } \\
\hline & 50,98 & 0,319 & 3,29 \\
\hline \multicolumn{2}{|c|}{$\left.\mathrm{Na}_{2} \mathrm{O}^{1}\right)($ Anm.S.547) 6,03 } & $0,094\}$ & \\
\hline $\mathrm{K}_{2} \mathrm{O}$ & 0,35 & $0,004\}$ & 1,01 \\
\hline $\mathrm{SO}_{3}$ & 30,96 & 0,387 & 4,00 \\
\hline $\mathrm{H}_{2} \mathrm{O}$ unter $103^{0}$ & 0,12 & & \\
\hline$I_{2} \mathrm{O}$ über $105^{0}$ & 11,03 & 0,613 & 6,33 \\
\hline $\mathrm{As}_{2} \mathrm{O}_{5}$ & 0,20 & & \\
\hline $\mathrm{SiO}_{2}$ & 0,23 & & \\
\hline \multirow[t]{2}{*}{$\mathrm{CaO}$} & 0,04 & & \\
\hline & 99,94 & & \\
\hline
\end{tabular}


Das Verhältniss von $\mathrm{Fe}_{2} \mathrm{O}_{3}: \mathrm{Na}_{2} \mathrm{O}: \mathrm{SO}_{3}: \mathrm{H}_{2} \mathrm{O}$ ist augenscheinlich $3: 1: 4: 6$, wie in dem gewöhnlichen Kaliumjarosit. Der geringe Ueberschuss von $\mathrm{Fe}_{2} \mathrm{O}_{3}$ und $\mathrm{H}_{2} \mathrm{O}$ ist wohl auf Kosten von etwas Eisenhydroxyd zu setzen, vielleicht sind auch die dunklen Einschlüsse theilweise daran schuld; ferner sind Spuren eines Arsenats und eines Silicats vorhanden. Wird der Ueberschuss an $\mathrm{Fe}_{2} \mathrm{O}_{3}$ und $\mathrm{H}_{2} \mathrm{O}$ als Verunreinigung abgerechnet, so ergiebt sich, dass $94 \%$ des analysirten Materials als reiner Natrojarosit angesehen werden müssen:

\begin{tabular}{|c|c|c|c|}
\hline & $\begin{array}{l}\text { ach Abzug d. } \\
\text { erunreinig.: }\end{array}$ & & $\begin{array}{l}\text { berechnet n. d. Formel: } \\
\quad \mathrm{Na}_{2} \mathrm{Fe}_{6}(\mathrm{OH})_{12}\left(\mathrm{SO}_{4}\right)_{4}\end{array}$ \\
\hline $\mathrm{Fe}_{2} \mathrm{O}_{3}$ & 46,43 oder & 49,39 & 49,49 \\
\hline $\mathrm{Na}_{2} \mathrm{O}$ & 5,81 & 6,18 & 6,39 \\
\hline $\mathrm{K}_{2} \mathrm{O}$ & 0,35 & 0,37 & - \\
\hline $\mathrm{SO}_{3}$ & 30,96 & 32,94 & 32,99 \\
\hline $\mathrm{H}_{2} \mathrm{O}$ & 10,45 & 11,12 & 11,13 \\
\hline & 94,00 & 100,00 & 100,00 \\
\hline
\end{tabular}

Die Anwesenheit von 6\% Verunreinigungen in einem Krystallpulver, wie das analysirte, kann nicht verwundern, wenn man bedenkt, dass unter Umständen fast 2500000 Kryställchen nöthig sind, um ein Gramm Substanz zu erhalten. Diese Schätzung beruht auf der Annahme, dass die Krystalle einen Durchmesser von $0,1 \mathrm{~mm}$ und eine Dicke von $0,02 \mathrm{~mm}$ haben, was sicherlich noch über ihre mittlere Grösse hinausgeht.

Unter den Stücken von Cook's Peak in New Mexico, welche Herr Porter eingesandt hatte, fanden sich einige Massen von etwas fester verkitteten Aggregaten winziger Kryställchen eines Minerals aus der Jarositgruppe. Die Stücke sind von braungelber Farbe und haben stellenweise das glitzernde Aussehen eines Glimmerschiefers. Auch hat es den Anschein, als ob dieselben einem Drucke unterworfen worden wären und zugleich eine gewisse Gleitung erfahren hätten. Das Material ist ziemlich leicht zu zerdrücken, und das Pulver zeigt unter dem Mikroskope alle Eigenschaften des oben beschriebenen Jarosits. Nls Begleiter finden sich ein wenig Limonit und Quarz; ganz reines Material zur Analyse konnte nicht erhalten werden. Es wurde daher eine Partialanalyse mit folgendem Resultate ausgeführt:

$$
\mathrm{Fe}_{2} \mathrm{O}_{3} 55,60, \mathrm{Na}_{2} \mathrm{O} 4,49, \mathrm{~K}_{2} \mathrm{O} \text { 0,77, } \mathrm{PbO} 0,96 \text {. }
$$

$\mathrm{SO}_{3}$ und $\mathrm{H}_{2} \mathrm{O}$ wurden nachgewiesen, aber nicht bestimmt; das Resultat ist hinlänglich genügend, um das Mineral als Natrojarosit festzulegen.

1) Nach dem völligen Glühen des Minerals wurden 0,22\% Natron nicht von heissem Wasser ausgezogen und mögen wohl einem Feldspathe oder sonstigen fremden Minerale angehören. Es wurden deshalb nur 5,81\% dem Jarosit zugerechnet und zur Berechnung der Formel benutzt. 


\section{Plumbojarosit.}

Das Material stammt von Cook's Peak in New Mexico und bildet ein schimmerndes krystallinisches Pulver oder lose zusammenhängende Massen, welche zwischen den Fingern leicht zerrieben werden können. Die Krystalle sind sehr symmetrisch und genau gleich denen des Natrojarosits, Fig. 1, obschon für gewöhnlich nur das eine Rhomboëder $r$ vorhanden ist. Sie sind im Mittel eine Kleinigkeit schmäler und etwas dünner als jene des Natrojarosits. Es wurden eine Anzahl Krystalle auf dem Reflexionsgoniometer gemessen, wobei die Hauptschwierigkeit mehr durch den vicinalen Charakter der Flächen, als durch deren Kleinheit bedingt wird.

Fig. 2.

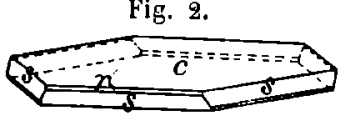

Ein ungewöhnlich grosser Krystall von $0,28 \mathrm{~mm}$ Breite und $0,015 \mathrm{~mm}$ Dicke zeigte die Entwickelung wie Fig. 2, welche ungewöhnlich ist, da im Allgemeinen stets $r\{10 T 1\}$ und nicht $s\{02 \overline{2} 1\}$ das vorherrschende Rhomboëder ist. Glücklicherweise war der Krystall so vortheilhaft an einer Wachsspitze angeheftet, dass Messungen von $s: s$ in drei rhomboëdrischen Zonen möglich waren. Die Resultate von fünf Messungen von $s: s$ über die obere und untere Polkante variiren zwischen $109^{\circ} 5^{\prime}$ und $109030^{\prime}$, im Mittel $109^{0} 16^{\prime}$, sechs Messungen über die Mittelkante variiren zwischen $70^{\circ} 10^{\prime}$ und $710^{\circ} 0^{\prime}$, im Mittel $70^{\circ} 36^{\prime}$. Das Mittel der beiden supplementären Werthe ist $s: s=(2 \overline{2} 01):(02 \overline{2} 1)=$ $109^{\circ} 20^{\prime}$, welcher Werth als Fundamentalwinkel genommen wurde. Daraus folgt das Axenverhältniss $a: c=1: 1,216$.

Die Basis ist an diesem Krystalle eine Vicinalfläche, weshalb auch keine brauchbaren Messungen für $e: s$ erhalten wurden. Dagegen wurde an einer Anzahl anderer Krystalle $c: r$ mit wechselndem Resultate gemessen, eine Folge der unsicheren Reflexe der Basis. Vier Messungen von $c: r$, welche als von den besten Reflexen erhalten bezeichnet werden müssen, schwanken zwischen $54015^{\prime}$ und $54^{0} 44^{\prime}$, im Mittel $54^{0} 30^{\prime}$, während der berechnete Winkel $e: r(0001):(10 T 1)=54^{0} 32^{\prime}$ ist. Es kann daher angenommen werden, dass das Axenverhältniss ziemlich exact ist. Der berechnete Werth von $r: r^{\prime}(10 T 1):(T 101)=89^{\circ} 42^{\prime}$.

Die Krystalle zeigen normale optische Eigenschaften und negative Doppelbrechung. Da die Krystalle im Mittel dünner sind als jene des Natrojarosits, so konnte nur ganz selten und mit den stärksten Vergrösserungen der Beginn des ersten Interferenzringes beobachtet werden. Einzelkrystalie zeigten unter dem Mikroskope in durchfallenden Lichte eine goldgelbe Farbe, während eine grössere Menge von Krystallen als dunkelbraunes, glänzendes Pulver erscheint, dunkler als das des Natrojarosits.

Die Analyse wurde mit dem bestmöglichen Materiale (spec. Gewicht 3,665 bei $30^{\circ} \mathrm{C}$.) ausgeführt. Das Resultat war ein überraschendes und gänz- 
lich unvorhergesehenes, indem sich ergab, dass dieser Jarosit an Stelle der Alkalien Blei enthält. Die gefundenen Zahlen (IIillebrand) sind:

$\begin{array}{lccccccc} & \text { I. } & \text { II. } & \text { III. } & \text { IV. } & \text { Mittel: } & \text { Verhältn.: } \\ \mathrm{Fe}_{2} \mathrm{O}_{3} & 42,36 & 42,38 & - & - & 42,37 & 0,265 & \\ \mathrm{Al}_{2} \mathrm{O}_{3} \text { ?1) } & 0,12 & 0,08 & \mathbf{0 , 1 1} & - & 0,10 & \mathbf{0 , 0 0 1} & 3,15 \\ \mathrm{PbO} & 19,69 & 19,99 & \mathbf{1 9 , 8 9} & \mathbf{1 9 , 7 9} & \mathbf{1 9 , 8 4} & \mathbf{0 , 0 8 9} & 1,05 \\ \mathrm{~K}_{2} \mathrm{O} & 0,17 & - & - & - & 0,17 & - & - \\ \mathrm{Na}_{2} \mathrm{O} & \left.0,211^{2}\right) & - & - & - & 0,212 & - & - \\ \mathrm{SO}_{3} & 27,05 & 27,07 & - & - & 27,06 & 0,338 & 4,00 \\ \mathrm{H}_{2} \mathrm{O} \text { unter } 1050 & 0,02 & - & - & - & 0,02 & - & - \\ \mathrm{H}_{2} \mathrm{O} \text { über } 105^{0} & 9,59 & 9,49 & - & - & 9,54 & 0,530 & 6,27 \\ \mathrm{SiO}_{2} & 0,56 & 0,51 & 0,47 & - & 0,51 & - & - \\ \mathrm{CuO} & 0,27 & 0,27 & - & - & 0,27 & - & - \\ \mathrm{CaO} & 0,05 & - & - & - & 0,05 & - & - \\ \mathrm{MgO} & 0,01 & - & - & - & 0,01 & - & -\end{array}$

Das Verhältniss von $\mathrm{Fe}_{2} \mathrm{O}_{3}: \mathrm{PbO}: \mathrm{SO}_{3}: \mathrm{H}_{2} \mathrm{O}$ ist sehr nahe $3: 1: 4: 6$, entspricht also dem Jarosit; der geringe Ueberschuss an $\mathrm{Fe}_{2} \mathrm{O}_{3}, \mathrm{H}_{2} \mathrm{O}$ und $\mathrm{PbO}+$ Alkalien kann wohl auf lechnung von Verunreinigungen gesetzt werden, theilweise Fisenhydroxyd und theilweise ein Bleisalz, vielleicht ein lüsliches Silicat, wie sich aus der vollständigen Lüslichkeit der Kieselsäure in Säuren ergiebt. Wird das Verhältniss genau gleich $3: 1: 4: 6$ gesetzt, so sind $4,36 \%$ Verunreinigungen zugegen, und die verbleibenden $95,64 \%$ können als reiner Plumbojarosit angesehen werden, wie folgt:

\begin{tabular}{|c|c|c|c|}
\hline \multirow[b]{2}{*}{$\mathrm{Fe}_{e_{2}} \mathrm{O}_{3}$} & \multicolumn{3}{|c|}{$\begin{array}{c}\text { Berechn. für } \\
\left.\mathrm{PbF} \mathrm{C}_{t}(\mathrm{OH})_{1 !}: \mathrm{SO}_{4}\right)_{4}\end{array}$} \\
\hline & 40,59 & oder & 42,44 \\
\hline $\mathrm{PbO}$ & 18,86 & & 19,72 \\
\hline $\mathrm{SO}_{3}$ & 27,06 & & 28,29 \\
\hline $\mathrm{H}_{2} \mathrm{O}$ & 9,13 & & 9,55 \\
\hline & 95,64 & & 100,00 \\
\hline
\end{tabular}

Da ungeführ 2500000 Krystalle von Natrojarosit für ein Gramm Substanz erforderlich sind, so sind sicherlich deren vier Millionen für ein Gramm Plumbojarosit nüthig, denn diese sind, obwohl von etwas höherem spec. Gewicht, doch bedeutend dünner. Es ist daher eine Verunreinigung von $4,5 \%$ in einem solchen krystallinischen Producte nicht verwunderlich.

1) Die Gegenwart von Thonerde wurde nicht genau geprüft. Die gegebenen Zahlen sind die Differenzen zwischen den verschiedenen Wägungen des Ammoniumniederschlages und jenen des in demselben enthaltenen Eisenoxyds, bestimmt durch Permanganat nach Reduclion mit Schwefelwasserstofr.

2) Wahrscheinlich elwas zu hoch. 


\section{Jarosit und Alunit.}

Wie aus den bisher bekannten Analysen ersichtlich, ist das darin bestimmte Alkali stets Kalium, obwohl auch etwas Natrium zuweilen vorhanden ist. Die Formeln für die beiden Mineralien sind daher:

$$
\begin{aligned}
& \mathrm{K}_{2} \mathrm{O} .3 \mathrm{Fe}_{2} \mathrm{O}_{3} .4 \mathrm{SO}_{3} .6 \mathrm{H}_{2} \mathrm{O} \text { und } \\
& \mathrm{K}_{2} \mathrm{O} .3 \mathrm{Al}_{2} \mathrm{O}_{3} .4 \mathrm{SO}_{3} .6 \mathrm{H}_{2} \mathrm{O}
\end{aligned}
$$

was auf verschiedene Art, wie wir noch sehen werden, ausgedrückt werden kann.

Ein Mineral, das unserem Natrojarosit entspricht, obwohl es eine kleine Quantität Kalium enthält, wurde von W. P. Headden ${ }^{1}$ ) beschrieben. Dasselbe, aus der Buxton-Mine, Lawrence Co., Süd-Dakota, stammend, bildet Blättchen, Combinationen eines Rhomboëders mit der Basis; das analysirte Material war ebenfalls nicht ganz rein, indem Quarz und etwas $\mathrm{As}_{2} \mathrm{O}_{5}$ angegeben werden. Letzteres gehört augenscheinlich nicht dem Jarosit an und kann vielleicht herrühren von etwas beigemengtem Skorodit $\mathrm{Fe} \mathrm{AsO} \mathrm{O}_{4}$. $2 \mathrm{H}_{2} \mathrm{O}$; unter dieser Annahme künnen die Resultate II eadden's folgendermassen interpretirt werden:

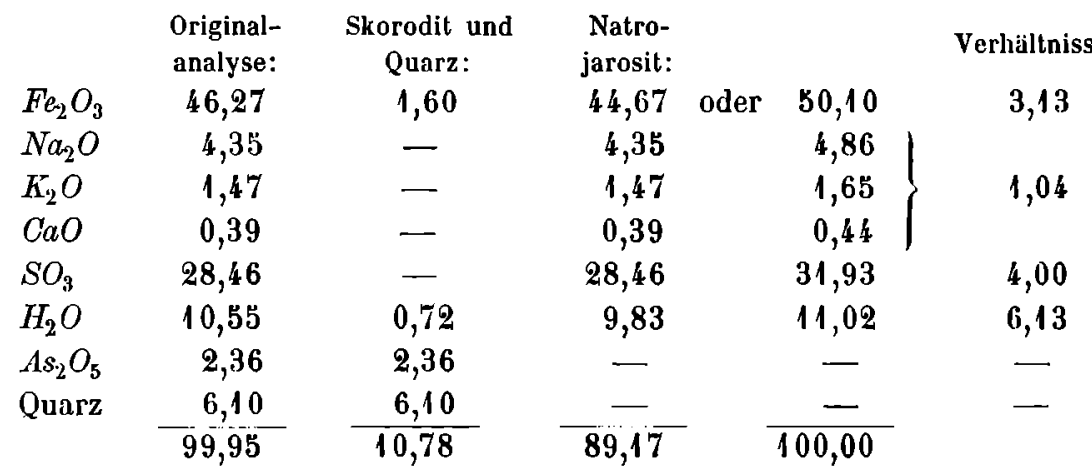

Werden also dergestait $4,68 \%$ Skorodit angenommen und mit dem Quarz in Abzug gebracht, so entspricht der Rest sehr gut dem Natrojarosit, indem das Molekularverhältniss ziemlich genau 3:1:4:6 wird.

Alunit mit betrüchtlichem Natrongehalte ist durch W. Cross ${ }^{2}$ ) von den Rosita IIills in Colorado und durch E. B. Hurlburt $\left.t^{3}\right)$ vom Red Mountain in Colorado bekannt geworden. Die Analysen beider Mineralien zeigen ungefähr gleiche Procente $\mathrm{K}_{2} \mathrm{O}$ und $\mathrm{Na}_{2} \mathrm{O}$ oder im Molekularverhältniss $\mathrm{K}_{2} \mathrm{O}$ : $\mathrm{Na}_{2} \mathrm{O}=4: 7$. Interessant ist, dass der Alunit vom Red Mountain in Colorado als krystallinisches Pulver vorkommt, dessen Krystāllchen genau

1) Amer. Journ. Sc. 1893, (3) 44, 24. Ref. diese Zeitschr. 25, 103.

2) Ebenda 1891 (3) 41, 472 . Ref. diese Zeitschr. 22, 421.

3) Ebenda 1894 (3) 48, 130. Hef. dicse Zeitschr. 26, 520. 
gleich denen des Natrojarosits und Plumbojarosits sind, nur dass die Krystalle etwas kleiner und weiss sind, unter dem Mikroskope aber farblos. Das Vorkommen von Natron in der Jarosit-Alunitgruppe ist daher vüllig in Uebereinstimmung mit früheren Beobachtungen; anders steht die Sache mit dem Blei. Es ist dies nach unserem Erachten der erste Fall, dass Blei isomorph mit den Alkalimetallen beolsachtet wird.

Vom chemischen Standpunkte aus ist das Interessanteste an dem neuen Minerale, dass es auf die Isomorphie von Kalium, Natrium und Blei hindeutet. Für gewöhnlich sind gerade Kalium und Natrium nicht isomorph, da die einfachsten Salze selten in denselben Formen krystallisiren. Obgleich $\mathrm{KCl}$ und $\mathrm{NaCl}$ beide in Würfeln krystallisiren, ist es keineswegs sicher, ob sie auch derselben Gruppe des isometrischen Systems gehören. Es ist z. B. durch die Aetzfiguren erwiesen, dass $\mathrm{KCl}$ gleich $\mathrm{NH}_{4} \mathrm{Cl}$ der plagi-

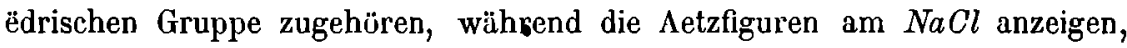
dass selbiges der normalen Gruppe angehürt. Fernerhin findet sich in Stassfurt der Sylvin neben dem Steinsalze auf demselben Handstücke auskrystallisirt, anstatt dass sie sich in isomorpher Mischung finden. Selbst in so complicirten Verbindungen, wie der Feldspath, krystallisiren die Kaliumund die Natriumverbindung als Orthoklas und Albit für sich leichter, denn als isomorphe Mischungen. Schliesslich hat Kalium eine starke Neigung Alaun zu bilden, was beim Natrium nicht zutrifft. Im Gegensatze zu diesem verschiedenen chemischen Verhalten haben wir in der Jarosit-Alunitgruppe nicht nur die Alkalimetalle $K a$ und $N a$, sondern, was noch bemerkenswerther erscheint, Blei in derselben Rolle in der Verbindung und Krystalle liefernd, welche in greradezu überraschender Weise in ihren physikalischen Eigenschaften einander gleichen. Wir künnen für den Augenblick keinen anderen Grund für diesen Fall der Isomorphie finden, als den, dass der Einfluss der Alkalien und des Bleies gegenüber den anderen Bestandtheilen so verschwindend ist, dass in dem grossen Moleküle die letzteren durch den Effect ihrer Masse die Krystallform bestimmen.

Der Alunit vom Red Mountain, den Hurlburt beschrieben hat, ist im mineralogischen Laboratorium der Sheffield-Iochschule untersucht worden, und es hat sich ergeben, dass das Wasser erst bei ziemlich hoher Temperatur ausgetrieben wird, dass es also nur als Hydroxyl und nicht als Krystallwasser zugegen sein kann. Daraus aber ergab sich, dass die scheinbar complicirte Formel des Minerals, welche dem Verhïltniss $\mathrm{Al}_{2} \mathrm{O}_{3}$ : $\mathrm{K}_{2} \mathrm{O}: \mathrm{SiO}_{3}: \mathrm{H}_{2} \mathrm{O}=3: 1: 4: 6$ entspricht, ganz bedeutend vereinfacht werden kann, nämlich zu $K\left[A l(\mathrm{OH})_{2}\right]_{3}\left[\mathrm{SO}_{4}\right]_{2}$.

Es wird jedoch durch gegenwärtige Untersuchung angezeigt erscheinen, die obige einfache Formel zu verlassen und diejenige mit doppelter Atomzahl anzunehmen, welche die Isomorphie zwischen $K a, N a$ und $P b$ zum 
Ausdrucke bringen lässt. Die Formeln der Mineralien würden sich demgemäss wie folgt gestalten:

$\begin{array}{lcrr}\text { Alunit } & \mathrm{K}_{2}\left[\mathrm{Al}(\mathrm{OH})_{2}\right]_{6}\left[\mathrm{SO}_{4}\right]_{4} & \text { oder } & \mathrm{K}_{2} \mathrm{Al}_{6}[\mathrm{OH}]_{12}\left[\mathrm{SO}_{4}\right]_{4} \\ \text { Natroalunit } & \mathrm{Na} a_{2}\left[\mathrm{Al}(\mathrm{OH})_{2}\right]_{6}\left[\mathrm{SO}_{4}\right]_{4} & \mathrm{Na}_{2} \mathrm{Al} l_{6}[\mathrm{OH}]_{12}\left[\mathrm{SO}_{4}\right]_{4} \\ \text { Jarosit } & \mathrm{K}_{2}\left[\mathrm{Fe}(\mathrm{OH})_{2}\right]_{6}\left[\mathrm{SO}_{4}\right]_{4} & \mathrm{~K}_{2} \mathrm{Fe}_{6}[\mathrm{OH}]_{12}\left[\mathrm{SO}_{4}\right]_{4} \\ \text { Natrojarosit } & \mathrm{Na} a_{2}\left[\mathrm{Fe}(\mathrm{OH})_{2}\right]_{6}\left[\mathrm{SO}_{4}\right]_{4} & \mathrm{Na}_{2} \mathrm{Fe}_{6}[\mathrm{OH}]_{12}\left[\mathrm{SO}_{4}\right]_{4} \\ \text { Plumbojarosit } & \mathrm{Pb}\left[\mathrm{Fe}(\mathrm{OH})_{2}\right]_{6}\left[\mathrm{SO}_{4}\right]_{4} & \mathrm{PbFe}_{6}[\mathrm{OH}]_{12}\left[\mathrm{SO}_{4}\right]_{4}\end{array}$

Bei der Bleiverbindung ist ein Atom Blei und in den übrigen zwei Atome Kalium oder Natrium combinirt mit einem Moleküle, bestehend aus 50 anderen Atomen. Es ist daher wohl begreiflich, dass der Complex von 50 Atomen zur Rechten von $\mathrm{Ka}_{2}, \mathrm{Na}_{2}$ und $\mathrm{Pb}$ in obigen Formeln die Krystallisation durch den Einfluss des Masseneffectes beherrscht und einen Isomorphismus bedingt zwischen so upgleichen Elementen wie Natrium, Kalium und Blei.

Die oben angenommene verdoppelte Formel kann natürlich wieder in mannigfacher Weise weiter in andere aufgelüst werden, von denen die folgenden die einfachsten und am meisten befriedigendsten sind:

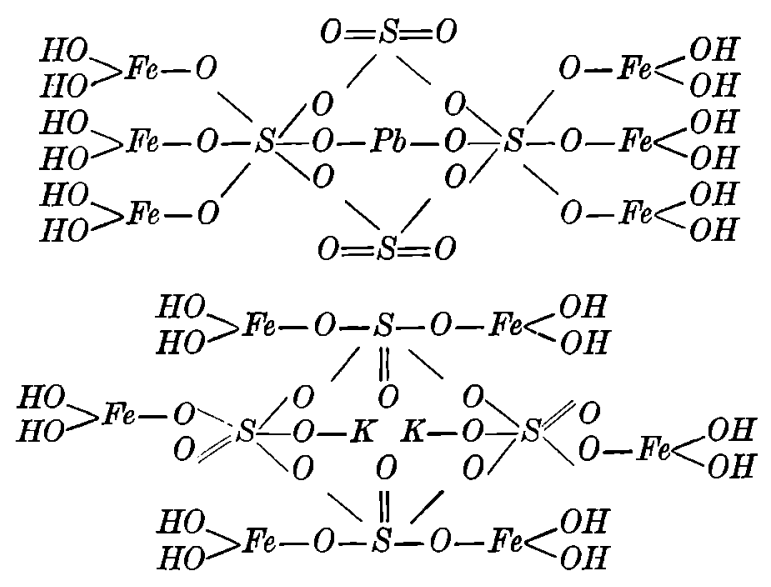

Bemerkenswerth ist, dass, obgleich die Rolle des $K_{2}, N a_{2}$ und $\mathrm{Pb}$ in den Alunit-Jarosit-Molekülen eine ganz untergeordnete ist, die Substitution von $\mathrm{Na}_{2}$ für $K_{2}$ dennoch begleitet wird von einer ganz auffallenden Veränderung der Krystallwinkel, welche in der That grösser ist, als sie im Allgemeinen bei isomorphen Vertretungen beobachtet wird. Dass Alunit und Jarosit, welche Kalium enthalten, in ihren Winkeln ziemlich gleich sein werden, war zu crwarten, da auch die Krystalle von Korund und Ilämatit überraschend gleich sind, wie folgender Vergleich ergiebt: 


$\begin{array}{lccrr} & \text { Axenlänge: } & r: r^{\prime} & c: r^{\prime} \\ \text { Korund } \mathrm{Al}_{2} \mathrm{O}_{3} & 1,3630 & 9^{\circ} 56^{\prime} & 57034^{\prime} \\ \text { IIÏimatit } \mathrm{Fe}_{2} \mathrm{O}_{3} & 1,3656 & 94 & 0 & 5737\end{array}$

Die Beziehungen der Mineralien der Alunit-Jarosit-Gruppe sind folgende:

$\begin{array}{lcccc} & \text { Axenlänge: } & r: r^{\prime} & c: r & \text { Doppelbrechung: } \\ \text { Alunit } & 1,252 & 90^{\circ} 50^{\prime} & 55^{0} 192^{\prime} & \text { positiv } \\ \text { Jarosit } & 1,245 & 9045 & 5516 & \text { negativ } \\ \text { Natrojarosit } & 1,104 & 8554 & 5153 & \text { negativ } \\ \text { Plumbojarosit } & 1,216 & 8942 & 5432 & \text { negativ }\end{array}$

Aus dieser Zusammenstellung ist ersichtlich, dass die Substitution von Natrium für Kalium im Jarosit eine grüssere Winkeländerung hervorgerufen hat, als die des Bleies an Stelle von Kalium.

Die drei Mineralien Natrojarosit, Plumbojarosit und $N a-K$-Alunit vom Red Mountain erscheinen zusammen unter dem Mikroskope praktisch völlig identisch und illustriren sehr schön einerseits die Isomorphie von Aluminium und Eisen und andererseits die von $K a, N a$ und Blei. Die drei Substanzen müssen wohl unter ganz ähnlichen Bedingungen entstanden sein, und es ist wahrscheinlich, dass sie Solfatarenproducte darstellen, welche unter der combinirten Wirkung von Ilitze und Druck entstanden sind. Da sie nur sehr schwer löslich sind, so haben sie sich, wie so manche Präcipitate, als feines krystallinisches Pulver abgeschieden.

Die drei Substanzen verhalten sich beim Erhitzen im geschlossenen Rohre vüllig gieich, bei mässigem Erhitzen erleiden sie keine Zersetzung, bei höher gesteigerter Temperatur zersetzen sich die Krystalle und zerfallen zu einem feinen Pulver oder Staub, welcher durch die entweichenden Dämpfe weitergeführt und erst in betrïchtlicher Entfernung im Rohre wieder abgesetzt wird. Ausser Wasser werden reichlich $\mathrm{SO}_{2}$ und $\mathrm{SO}_{3}$ abgegeben. Beim Natrojarosit ergab sich, und dies wird zweifelsohne auch für den $\mathrm{Na}$-K-Jarosit gelten, dass sich nach dem Glühen ein Viertel des Schwefelsäureradicals an Alkali gebunden findet und mit Wasser ausgezogen werden kann, während der Plumbojarosit beim Glühen alle Schwefelsäure abgiebt, zweifelsohne, weil das vorhandene Eisenoxyd zur Zersetzung alles Bleisulfates dient, das sich etwa bilden künnte. Erhitzt man Anglesit $\mathrm{PbSO}_{4}$ für sich im geschlossenen Rohre, so erleidet er keine Zersetzung, wird derselbe aber mit Limonit fein zerrieben und erhitzt, so wird sauer reagirendes Wasser abgegeben. Fein gepulverter Natrojarosit und Plumbojarosit sind langsam aber vollständig in kochender Salzsüure lüslich, Plumbojarosit giebt mit Soda auf Kohle Kügelchen von Blei und einen Bleibeschlag.

Es schien den Verfn. am besten, die neuen Verbindungen als Natrojarosit und Plumbojarosit zu bezeichnen, da diese Namen die Beziehungen zu wohlbckannten Mineralien ausdrücken. Andere Glieder dieser Gruppe 
554 W. F. Hillebrand u. S. I. Penfield. Beitr. z. Kenntn. der Alunit-Jarositkrystalle.

werden zweifelsohne noch gefunden werden, und der Name Natroalunit soll zur Bezeichnung der auf Seite 534 erwähnten Varietät des Alunits von Colorado dienen, in welchem das Verhältniss von Natron zu Kali $=7: 4$ ist. Es ist sehr wahrscheinlich, dass eine Reihe von Alunit-Jarosit-Verbindungen sich auch künstlich darstellen lassen wird.

Schliesslich gedenken die Verff. dankbarst dẹ Herren Turner und Porter, welche die Verff. auf diese interessanten Verbindungen hingewiesen haben.

Laborat. der U. S. Geolog. Survey, Washington.

Laborat. der Sheffield Sc. School, New IIaven. 